\title{
Activités de l'uranium et du radium dans des échantillons d'eau naturelle au Sahara marocain
}

\author{
0.-K. Hakam, A. Choukri*, H. Bounouira et M. Al Ibrahimi \\ Laboratoire de physique de la matière et rayonnement, Equipe de physique et \\ techniques nucléaires, Faculté des sciences, P.B. 133, Kénitra 14000, Maroc
}

(Reçu le 02 Mai 2006, accepté le 17 Août 2006)

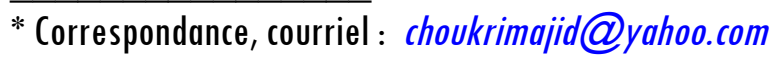

\section{Résumé}

Dans ce travail, on donne les résultats préliminaires de mesure des activités des principaux radio-isotopes de l'uranium et du radium $\left({ }^{238} \mathrm{U},{ }^{234} \mathrm{U},{ }^{226} \mathrm{Ra},{ }^{228} \mathrm{Ra}\right.$ et de ${ }^{228} \mathrm{Th}$ ) et des rapports d'activité de ces radio-isotopes $\left({ }^{234} \mathrm{U} /{ }^{238} \mathrm{U},{ }^{228} \mathrm{Ra} /{ }^{226} \mathrm{Ra},{ }^{228} \mathrm{Th} /{ }^{228} \mathrm{Ra},{ }^{226} \mathrm{Ra} /{ }^{238} \mathrm{U}\right)$ obtenus pour des échantillons d'eau de 8 puits, 5 forages, 2 sources et 3 eaux de robinet échantillonnés dans les régions des villes de Laayoune et Es-Smara au Sahara marocain. Ces échantillons d'eau naturelle proviennent de sources placées dans une région de désert riche en phosphate. Sauf pour un forage dont l'eau est non utilisée par la population, le calcul de doses équivalentes aux activités maximales pour chaque isotope montre que toutes les activités mesurées sont inférieures aux activités maximales recommandées par la Commission Internationale de Protection des Rayonnements et elles ne présentent pas de risque pour la santé de la population. Les activités et les rapports d'activité mesurés sont comparables à ceux que l'on trouve habituellement dans d'autres régions du Maroc ou du monde.

Mots-clés : uranium, radium, thorium, phosphate, le Sahara marocain, eau naturelle.

\section{Abstract}

Measure of uranium and radium activities in some natural water samples from Moroccan Sahara

The preliminary results of ${ }^{238} \mathrm{U},{ }^{234} \mathrm{U},{ }^{226} \mathrm{Ra},{ }^{228} \mathrm{Ra}$ and ${ }^{228} \mathrm{Th}$ activities and ${ }^{20}{ }^{234} \mathrm{U} /{ }^{238} \mathrm{U}$, ${ }^{228} \mathrm{Ra} /{ }^{226} \mathrm{Ra},{ }^{228} \mathrm{Th} /{ }^{228} \mathrm{Ra}$ and ${ }^{226} \mathrm{Ra} /{ }^{238} \mathrm{U}$ activity ratios obtained for 8 wells, 5 drillings, 2 springs and 3 tap water samples collected in Laayoune and Es-Smara regions in the Moroccan Sahara are given in this work. The analyzed natural water samples have been 
collected from sources situated in a desert region rich in phosphates. Except for the one water drilling sample no used by population, the calculation of equivalent doses to the maximal activities for each isotope show that all measured activities are inferior to the maximum contaminant levels recommended by the International Commission of Radioprotection and they don't present any risk for population health. The obtained results are comparable to those already found in other regions in Morocco or in the world.

Keywords : uranium, radium, thorium, phosphate, Moroccan Sahara, natural water.

\section{Introduction}

La radioactivité qu'on trouve dans notre environnement, provient essentiellement des radioéléments des familles radioactives naturelles. La répartition de ces radioéléments dans les différents milieux dépend de leur état physico-chimique et de leur comportement géochimique en fonction des conditions naturelles régnantes.

La présence et le comportement des radioéléments dans les eaux naturelles de différentes sources ont été étudiés à travers le monde [1-13].

Le comportement des éléments des séries de l'uranium et du thorium dans ces eaux a un grand intérêt en géochimie, pour l'exploration de l'uranium et pour la santé.

Les concentrations et les activités des radio-isotopes de l'uranium et du radium ont été utilisées pour étudier certains phénomènes naturels et suivre des processus d'altération de certaines formations géologiques ou de transport de certaines matières par les courants d'eau. Elles ont été utilisées également pour étudier l'interaction eaux continentales - eau de mer.

La radioactivité dans les eaux naturelles provient, essentiellement, de radionucléides primordiaux dans l'air ou dans les sols ( $\left({ }^{40} \mathrm{~K}\right.$ et les séries $4 n$ et $\left.4 n+2\right)$. Des radionucléides dus à une pollution radioactive à partir des formations géologiques riches en radioéléments, des mines d'uranium, ou à partir des rejets de déchets radioactifs ou d'une utilisation abusive de fertilisants agricoles, peuvent être trouvés dans les eaux naturelles [1-5]. Des nucléides cosmogéniques, tels que ${ }^{3} \mathrm{H}$ et ${ }^{3} \mathrm{Be}$ sont souvent associés à ces eaux à travers l'eau de pluie.

La connaissance des concentrations de l'uranium et du radium dans les eaux potables est importante parce qu'une fraction de l'uranium et du radium absorbés est déposée dans le corps humain, introduisant ainsi une dose radioactive interne équivalente.

Les concentrations en éléments radioactifs naturels dans les eaux profondes et les eaux de surface, peuvent être reliées aux conditions physico-chimiques de l'eau et à son environnement géologique. Cette radioactivité est essentiellement due à l'uranium, au 
thorium ainsi qu'à leurs descendants, dont l'eau s'est chargée lors de son passage au travers des roches plutoniques ou métamorphiques profondes. Par exemple, les sources les plus radioactives sont fréquemment les sources carbo-gazeuses ou chlorurées dont les caractéristiques chimiques favorisent la solubilisation des éléments lourds comme l'uranium et le radium.

Dans ce travail, on donne des résultats obtenus sur quelques échantillons d'eau naturelle prélevés à partir des sources naturelles situées dans une région de désert riche en phosphate au Sud du Maroc. Ces résultats permettront de comparer les activités des principaux radio-isotopes de l'uranium et du radium $\left({ }^{238} \mathrm{U},{ }^{234} \mathrm{U},{ }^{226} \mathrm{Ra},{ }^{228} \mathrm{Ra}\right.$ et de ${ }^{228} \mathrm{Th}$ ) et des rapports d'activité de ces radio-isotopes $\left({ }^{234} \mathrm{U} /{ }^{238} \mathrm{U},{ }^{228} \mathrm{Ra} /{ }^{226} \mathrm{Ra},{ }^{228} \mathrm{Th} /{ }^{228} \mathrm{Ra},{ }^{226} \mathrm{Ra} /{ }^{238} \mathrm{U}\right)$ avec ceux trouvés dans d'autres sites marocains et internationaux; ce qui permettra d'orienter, dans le futur, d'autres campagnes d'échantillonnage dans le but d'établir une carte de distribution des radioéléments dans les eaux naturelles du Maroc.

\section{Echantillonnage et méthodologie}

\section{2-1. Région étudiée}

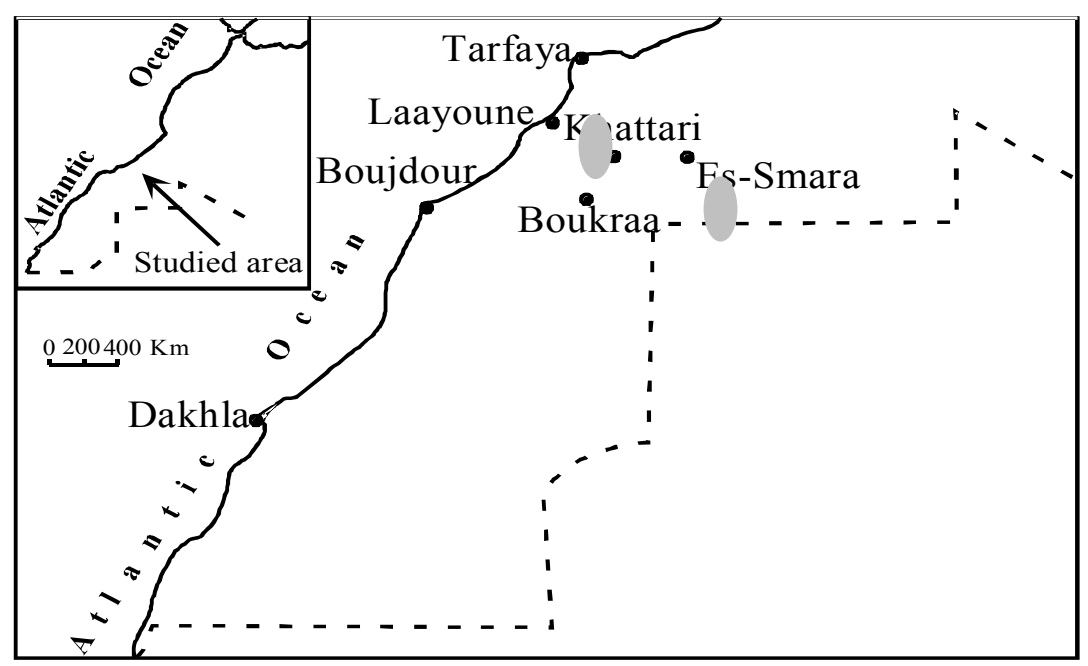

Figure 1 : Localisation des sites d'échantillonnage d'eau naturelle au Sahara marocain.

\section{2-2. Préparation de l'échantillon}

La technique d'échantillonnage consiste à prélever 20 litres d'eau dans des bouteilles hydrologiques. L'eau est acidifiée à $\mathrm{pH}=2$ avec l'acide chlorhydrique concentré; une quantité connue de traceurs $\left({ }^{232} \mathrm{U}-{ }^{228} \mathrm{Th}\right)$ est introduite afin de connaître le rendement 
global : récupération, traitement chimique et détection. Après homogénéisation, l'uranium et le thorium sont co-précipités avec les hydroxydes de fer formés par l'ajout de l'ammoniaque à $\mathrm{pH}=7$ dans le milieu. Les isotopes de radium $\left({ }^{226} \mathrm{Ra}\right.$ et ${ }^{228} \mathrm{Ra}$ ) sont coprécipités avec $\mathrm{BaSO}_{4}$ formé par ajout de $\mathrm{H}_{2} \mathrm{SO}_{4}$. La précipitation des hydroxydes de fer est faite avant la précipitation du baryum pour ne pas piéger l'uranium dans la matrice du $\mathrm{BaSO}_{4}$ qui est insoluble en milieu acide [6]. Le précipité de sulfate de baryum et des hydroxydes de fer est récupéré par décantation et filtration. Après, il faut séparer l'uranium et le thorium du radium. On sait que le ( $\mathrm{Ba}-\mathrm{Ra}_{3} \mathrm{SO}_{4}$ est insoluble en milieu acide, donc on attaque le précipité de sulfate de baryum et des hydroxydes de fer par l'acide concentré, l'uranium et le thorium sont solubles alors que le $(\mathrm{Ba}-\mathrm{Ra}) \mathrm{SO}_{4}$ insoluble est récupéré par centrifugation. Ce résidu est séché et pesé avant d'être mis dans un tube convenable pour le comptage dans le détecteur germanium puits. Le comptage est fait après que l'équilibre soit établi entre les isotopes du radium et leurs descendants, soit une période d'environ 20 à 30 jours qui correspond à peu près à 10 périodes du ${ }^{222} \mathrm{Rn}$, gaz responsable d'une rupture permanente de l'équilibre entre le radium et les autres descendants.

\section{2-3. Partie expérimentale}

La procédure de mesure, de comptage et de calcul utilisée est identique à celle décrite par les auteurs [6-8,14] pour la spectrométrie gamma et à celle décrite par Choukri et al. pour la spectrométrie alpha [15].

La technique utilisée consiste à prélever, dans des bidons en plastique, 5 litres d'eau pour une analyse unique de l'uranium, et 20 litres d'eau pour une analyse double de l'uranium et du radium. Après filtration sur des membranes de marque Millipore de porosité $0,4 \mu \mathrm{m}$ et de diamètre $147 \mathrm{~mm}$, l'échantillon d'eau est acidifié à $\mathrm{pH}=2$ à 3 avec $\mathrm{HNO}_{3}$ avant de rajouter une solution contenant des quantités connues des traceurs ${ }^{232} \mathrm{U}$ et ${ }^{228} \mathrm{Th}$ ) et du fer $(50 \mathrm{à} 80 \mathrm{mg}$ ), et une solution contenant 1 gramme de chlorure de baryum $\left(\mathrm{BaCl}_{2}\right)$. Les isotopes de l'uranium et du thorium sont co-précipités avec les hydroxydes de fer formés par l'ajout de l'ammoniaque à $\mathrm{pH}=7$ dans le milieu avant de précipiter le radium avec $\mathrm{BaSO}_{4}$ formé par l'ajout de $\mathrm{H}_{2} \mathrm{SO}_{4}$. Le précipité de sulfate de baryum et des hydroxydes de fer sont récupérés ensemble par décantation et filtration.

L'uranium et le thorium sont dissous avec les hydroxydes par l'ajout d'acide concentré, tandis que le radium est retenu dans le résidu de sulfate de baryum qui est insoluble dans le milieu acide. Ce résidu est lavé, séché et pesé avant d'être scellé dans un tube convenable pour le comptage dans le détecteur germanium puits à très bas bruit de fond. Le comptage est fait après 20 jours de scellage, temps nécessaire pour que l'équilibre soit établi entre les isotopes du radium et leurs descendants. Les activités des isotopes du radium sont mesurées à l'aide de ses descendants émetteurs gamma de courtes 
périodes $\left({ }^{214} \mathrm{~Pb}\right.$ et ${ }^{214} \mathrm{Bi}$ pour ${ }^{226} \mathrm{Ra}$ et ${ }^{228} \mathrm{Ac}$ pour $\left.{ }^{228} \mathrm{Ra}\right)$. Elles sont corrigées du rendement de précipitation du sulfate de baryum en tenant compte du facteur de correction du poids $(1,16)$ due à la formation de $\mathrm{BaSO}_{4}$ à partir de $\mathrm{BaCl}_{2}$. Les standards gamma utilisés pour I'inter-calibration sont N.B.S (National Bureau of Standard USA) 4363 et 4350B, et AIEA 375.

La détermination des activités des isotopes de l'uranium $\left({ }^{238} \mathrm{U}\right.$ et $\left.{ }^{234} \mathrm{U}\right)$ est effectuée par spectrométrie alpha en utilisant un détecteur à barrière de surface. Elle est précédée d'une séparation chimique poussée sur résine échangeuse d'ions selon la procédure chimique décrite par Choukri et al. [15].

Les activités des principaux radio-isotpes de l'uranium et du radium $\left({ }^{238} \mathrm{U},{ }^{234} \mathrm{U},{ }^{226} \mathrm{Ra},{ }^{228} \mathrm{Ra}\right.$ et de ${ }^{228} \mathrm{Th}$ ) et des rapports d'activité de ces radio-isotopes $\left({ }^{234} \mathrm{U} /{ }^{238} \mathrm{U},{ }^{228} \mathrm{Ra} /{ }^{226} \mathrm{Ra}\right.$, ${ }^{228} \mathrm{Th} /{ }^{228} \mathrm{Ra},{ }^{226} \mathrm{Ra} /{ }^{238} \mathrm{U}$ ) ont été mesurés dans des échantillons d'eau naturelle de 8 puits, 5 forages, 2 sources et 3 eaux de robinet.

\section{Résultats et discussion}

Les résultats préliminaires, obtenus dans ce travail, sont donnés dans le Tableau 1 .

Tableau 1 : Activités et rapports d'activités des radio-isotopes de U et Ra dans les échantillons d'eau analysés

\begin{tabular}{|c|c|c|c|c|c|c|c|c|c|c|}
\hline $\begin{array}{l}\text { Source } \\
\text { d' eau }\end{array}$ & Echantillon & $\begin{array}{l}{ }^{234} \mathbf{U} \\
\left(\mathrm{mBq}, \mathrm{L}^{-1}\right)\end{array}$ & $\begin{array}{l}{ }^{238} \mathrm{U} \\
\left(\mathrm{mBq}, \mathrm{L}^{-1}\right)\end{array}$ & ${ }^{234} \mathbf{U} /{ }^{238} \mathbf{U}$ & $\begin{array}{l}{ }^{226} \mathbf{R a} \\
\left(\mathrm{mBq}, \mathrm{L}^{-1}\right)\end{array}$ & $\begin{array}{l}{ }^{228} \mathbf{R a} \\
\left(\mathrm{mBq}, \mathrm{L}^{-1}\right)\end{array}$ & $\begin{array}{l}{ }^{228} \mathrm{Th} \\
\left(\mathrm{mBq}, \mathrm{L}^{-1}\right)\end{array}$ & $\begin{array}{l}{ }^{228} \mathrm{Ra} / \\
{ }^{226} \mathrm{Ra}\end{array}$ & $\begin{array}{l}{ }^{228} \mathrm{Th} / \\
{ }^{228} \mathrm{Ra}\end{array}$ & $\begin{array}{l}{ }^{226} \mathrm{Ra} / \\
{ }^{238} \mathrm{U}\end{array}$ \\
\hline \multicolumn{11}{|l|}{ Puits } \\
\hline Es_Smara & $\begin{array}{c}\text { SI } \\
\text { (Echgag) }\end{array}$ & $181 \pm 16$ & $114 \pm 10$ & $1,58 \pm 0,05$ & $80,0 \pm 0,5$ & $26,6 \pm 0,6$ & $5,3 \pm 0,2$ & 0,33 & 0,20 & 0,70 \\
\hline Laayoune & $\begin{array}{c}57 \\
\text { (Lamriate) }\end{array}$ & $23 \pm 2$ & $15 \pm 1$ & $1,44 \pm 0,06$ & $118,0 \pm 0,6$ & $60,0 \pm 0,8$ & $88,2 \pm 0,5$ & 0,51 & 1,47 & 7,52 \\
\hline Laayoune & $\begin{array}{c}\text { S8 } \\
\text { (Tarfaya) }\end{array}$ & $59 \pm 4$ & $31 \pm 2$ & $1,88 \pm 0,08$ & $297,0 \pm 1,4$ & $200,0 \pm 2,1$ & $16,0 \pm 0,2$ & 0,67 & 0,08 & 9,5 \\
\hline Laayoune & $\begin{array}{c}59 \\
\text { (Daoural) }\end{array}$ & $549 \pm 61$ & $187 \pm 21$ & $2,93 \pm 0,08$ & $42,0 \pm 0,5$ & $18,9 \pm 0,3$ & $2,60 \pm 0,04$ & 0,45 & 0,14 & 0,22 \\
\hline Laayoune & $\begin{array}{c}\text { S10 } \\
\text { (Daoura2) }\end{array}$ & $389+41$ & $124 \pm 13$ & $3,14 \pm 0,10$ & $132,0 \pm 0,4$ & $84,4 \pm 1,3$ & $16,9 \pm 0,2$ & 0,64 & 0,20 & 1,07 \\
\hline Laayoune & $\begin{array}{c}511 \\
(7 \mathrm{~km} \text { de } \\
\text { Tarfaya) } \\
\end{array}$ & $44 \pm 4$ & $42 \pm 4$ & $1,06 \pm 0,06$ & $114,0 \pm 0,8$ & $51,5 \pm 1,1$ & $11,3 \pm 0,2$ & 0,45 & 0,22 & 2,75 \\
\hline Laayoune & $\begin{array}{c}\text { S17 } \\
\text { (Foum Oued l) }\end{array}$ & & & & $38,0 \pm 0,2$ & $8,0 \pm 0,2$ & $5,8 \pm 0,2$ & 0,21 & 0,73 & \\
\hline Laayoune & $\begin{array}{c}518 \\
\text { (Foum Oued2) }\end{array}$ & - & - & _- & $31,0 \pm 0,5$ & $8,5 \pm 0,5$ & $4,4 \pm 0,2$ & 0,27 & 0,52 & - \\
\hline
\end{tabular}




\begin{tabular}{|c|c|c|c|c|c|c|c|c|c|c|}
\hline $\begin{array}{l}\text { Source } \\
\text { d' eau }\end{array}$ & Echantillon & $\begin{array}{l}{ }^{234} \mathbf{U} \\
\left(\mathrm{mBq}, \mathrm{L}^{-1}\right)\end{array}$ & $\begin{array}{l}{ }^{238} \mathbf{U} \\
\left(\mathrm{mBq}, \mathrm{L}^{-1}\right)\end{array}$ & ${ }^{234} \mathbf{U} /{ }^{238} \mathbf{U}$ & $\begin{array}{l}{ }^{226} \mathbf{R a} \\
\left(\mathrm{mBq}, \mathrm{L}^{-1}\right)\end{array}$ & $\begin{array}{l}{ }^{228} \mathbf{R a} \\
\left(\mathrm{mBq}, \mathrm{L}^{-1}\right)\end{array}$ & $\begin{array}{l}{ }^{228} \mathrm{Th} \\
\left(\mathrm{mBq}, \mathrm{L}^{-1}\right)\end{array}$ & $\begin{array}{l}{ }^{228} \mathrm{Ra} / \\
{ }^{226} \mathrm{Ra}\end{array}$ & $\begin{array}{l}{ }^{228} \mathrm{Th} / \\
{ }^{228} \mathrm{Ra}\end{array}$ & $\begin{array}{l}{ }^{226} \mathrm{Ra} / \\
{ }^{238} \mathrm{U}\end{array}$ \\
\hline \multicolumn{11}{|l|}{ Forages } \\
\hline Es_Smara & $\begin{array}{l}\text { S2 } \\
\text { (Khattari) }\end{array}$ & $2,7 \pm 0,2$ & $1,3 \pm 0,1$ & $2,11 \pm 0,29$ & $93,0 \pm 0,7$ & $227,0 \pm 2,0$ & $29,5 \pm 0,3$ & 2,44 & 0,13 & 72 \\
\hline Laayoune & $\begin{array}{l}\text { S6 } \\
\text { (L'aïne) }\end{array}$ & $7,1 \pm 0,4$ & $4,7 \pm 0,3$ & $1,49 \pm 0,09$ & $12851 \pm 7$ & $10689 \pm 10$ & $641 \pm 6$ & 0,83 & 0,06 & 2734 \\
\hline Laayoune & $\begin{array}{l}\text { SI2 } \\
\text { (Tah) }\end{array}$ & $33 \pm 2$ & $23 \pm 2$ & $1,42 \pm 0,08$ & $318,0 \pm 0,8$ & $47,1 \pm 2,2$ & $9,9 \pm 0,2$ & 0,15 & 0,21 & 13,83 \\
\hline Laayoune & $\begin{array}{l}\text { S13 } \\
\text { (Boukraal) }\end{array}$ & $154 \pm 14$ & $114 \pm 10$ & $1,36 \pm 0,04$ & $594 \pm 2$ & $142,0 \pm 1,6$ & $17,0 \pm 0,2$ & 0,24 & 0,12 & 5,23 \\
\hline Laayoune & $\begin{array}{l}\text { S16 } \\
\text { (Boukrau2) }\end{array}$ & . & $\cdots-1$ & & $44,0 \pm 0,4$ & $46,7 \pm 0,8$ & $8,9 \pm 0,2$ & 1,06 & 0,19 & \\
\hline \multicolumn{11}{|c|}{ Sources } \\
\hline Laayoune & $\begin{array}{l}\text { S14 } \\
\text { (Lamsaid) }\end{array}$ & $47 \pm 4$ & $33 \pm 3$ & $1,44 \pm 0,08$ & $502 \pm 2$ & $299,0 \pm 1,6$ & $41,9 \pm 0,3$ & 0,60 & 0,14 & 15,35 \\
\hline Laayoune & $\begin{array}{l}\text { S15 } \\
\text { (Tissgrade) }\end{array}$ & $47 \pm 5$ & $35 \pm 4$ & $1,33 \pm 0,09$ & $1030 \pm 1$ & $744,0 \pm 2,7$ & $81,8 \pm 0,4$ & 0,72 & 0,11 & 29,43 \\
\hline \multicolumn{11}{|c|}{$\begin{array}{l}\text { Eaux de } \\
\text { Robinet }\end{array}$} \\
\hline Es_Smara & $\begin{array}{l}\text { S3 } \\
\text { (Es-Smara) }\end{array}$ & & - & $2,30 \pm 0,44$ & $62,0 \pm 0,6$ & $137,0 \pm 1,4$ & $17,8 \pm 0,2$ & 2,21 & 0,13 & _- \\
\hline Es_Smara & $\begin{array}{l}\text { S4 } \\
\text { (Gwaiaze) }\end{array}$ & $58 \pm 5$ & $33 \pm 3$ & $1,78 \pm 0,10$ & $45,0 \pm 0,7$ & $30,5 \pm 0,9$ & $9,5 \pm 0,2$ & 0,68 & 0,31 & 1,38 \\
\hline Laayoune & $\begin{array}{l}55 \\
\text { (Laayoune) } \\
\end{array}$ & $41 \pm 4$ & $34 \pm 3$ & $1,20 \pm, 07$ & $34,0 \pm 0,5$ & $14,4 \pm 0,6$ & $4,6 \pm 0,2$ & 0,42 & 0,32 & 0,99 \\
\hline
\end{tabular}

Les résultats obtenus montrent que les activités de ${ }^{238} \mathrm{U}$ sont relativement plus hautes dans des échantillons d'eau de puits et celles du ${ }^{226} \mathrm{Ra}$ sont généralement plus hautes dans des échantillons d'eau de source. Cette tendance a été déjà observée dans nos travaux publiés précédemment [6-8].

A l'exception de l'échantillon provenant du forage 56 situé dans une région riche en phosphates où les activités de ${ }^{226} \mathrm{Ra},{ }^{228} \mathrm{Ra}$ et de ${ }^{228} \mathrm{Th}$ sont relativement élevées par rapport aux autres activités, les activités et les rapports d'activité obtenus pour le reste des échantillons sont comparables à ceux que l'on trouve habituellement au Maroc [6-9] ou dans le monde [1-5, 10-13].

Dans la phase dissoute, l'activité de ${ }^{234} \mathrm{U}$ est généralement en excès comparée à celle de ${ }^{238} \mathrm{U}$ aboutissant à un rapport d'activité ${ }^{234} \mathrm{U} /{ }^{238} \mathrm{U}$, généralement supérieur à l'unité [4]. Les rapports d'activité é ${ }^{234} \mathrm{U} /{ }^{238} \mathrm{U}$ mesurés confirment cette hypothèse, ils varient entre 1,06 et 3,14 et ils ne montrent pas de tendance de variation avec ${ }^{238} \mathrm{U}$. Ce résultat confirme ce qui a été déjà constaté par Hakam et al. $[8,9]$ dans des échantillons d'eaux de source minérales marocaines caractérisées par de faibles activités de ${ }^{238} \mathrm{U}$ et de hautes valeurs d'activités de ${ }^{226} \mathrm{Ra}$ et des rapports d'activités ${ }^{234} \mathrm{U} /{ }^{238} \mathrm{U}$. 
${ }^{228} \mathrm{Ra} /{ }^{226} \mathrm{Ra}$ donne le rapport d'activité entre l'isotope ${ }^{228} \mathrm{Ra}$ produit par ${ }^{232} \mathrm{Th}$ et ${ }^{226} \mathrm{Ra}$ produit par ${ }^{238} \mathrm{U}$. Les valeurs calculées pour ce rapport sont élevées à part trois échantillons (2 puits et 1 eau de robinet); elles varient dans un large intervalle entre 0,22 et 2734 .

Le rapport d'activitée ${ }^{228} \mathrm{Th} /{ }^{228} \mathrm{Ra}$, indiquant l'état d'équilibre entre le ${ }^{228} \mathrm{Ra}$ soluble et son descendant ${ }^{228} \mathrm{Th}$ insoluble dans les conditions naturelles, est, dans la plupart des cas, inférieur à 1 sauf pour l'échantillon d'eau de puits 57 où il est égal à l,47.

\section{Conclusion}

L'analyse radioactive de 8 échantillons d'eau de puits, 5 échantillons prélevés à partir de forages, 2 échantillons de sources et 3 échantillons d'eau de robinet prélevés dans les régions des villes de Laayoune et de Es-smara au Sahara marocain, nous a permis d'avoir une idée préliminaire sur la répartition des activités des principaux radio-isotopes de l'uranium et du radium dans ces sources d'eau. Les résultats obtenus permettront d'orienter les prochaines campagnes d'échantillonnage et contribueront à établir une carte de la répartition de la radioactivité naturelle dans les différentes sources d'eau naturelle au Maroc. Ils permettront également de combler le vide qui existe en ce qui concerne les données sur la radioactivité dans les eaux souterraines et les eaux de surface d'une manière générale en Afrique et dans le monde Arabe. Les activités mesurées sont comparables à celles déjà trouvées dans d'autres régions du monde.

En comparant ces résultats à ceux déjà utilisés par Hakam et al. [8] pour montrer que les activités mesurées dans les échantillons naturelles au Maroc ne dépassent pas les limites maximales fixées par l'ICRP, on peut conclure également que les activités mesurées dans ce travail ne dépassent pas aussi ces limites et par conséquent, elles ne présentent aucun risque sanitaire pour la population.

\section{Remerciements}

Ce travail est réalisé dans le partenariat de l'UFR "Faibles Radioactivités, Physique Mathématique et Environnement" et le Programme national PROTARS II ( $n^{\circ}$ P2I/60) du Maroc.

\section{Références}

[1] - M. Asikainen, H. Kahols "Anomalously High concentrations of uranium, radium and radon in water from drilled wells in the Helsinki region". Geochim. Cosmochi. Acta, 43 (1979) 1681-1686.

[2] - J. N. Andrews, R. L. Kay "The $U$ content and ${ }^{234} \mathrm{U} /{ }^{238} \mathrm{U}$ activity ratio of dissolved uranium in groundwater from some triassic sandstones in England." Isot. Geosci., 1 (1983) 101-117. 
[3] - N. Hussain, S. Krishnaswami "U-238 series radioactive disequilibrium in groundwaters: Implications to the origin of excess U-238 and fate of reactive pollutants." Geochim. Cosmochim. Acta, 44 (1980) 1287-1291.

[4] - Ivanovich, M. "Uranium series disequilibrium: Applications to earth, marine and environmental sciences ( ${ }^{\text {nd }}$ edition)." (1992), Cladendon Press, 0xford.

[5] - A. Mangini, C. Sonntag, G. Bertsch, E. Muller "Evidence for a higher natural uranium content in world rivers." Nature, 278 (1979) 337-339.

[6] - 0.-K. Hakam "Etude de la répartition des activités des radio-isotopes de l'uranium et du radium dans les eaux naturelles et du radon dans l'air des habitations au Maroc", Thèse d'Éfat, Univ. Med V, (2000) $212 \mathrm{p}$.

[7] - 0.-K. Hakam, A. Choukri, J.-L.Reyss, M. Lferde "Determination and comparison of uranium and radium isotopes activities and activity ratios in samples from natural water sources in Morocco." J. Environ. Radioactivity, 57 (2001) 175-189.

[8] - O.K. Hakam, A. Choukri, J.-L.Reyss, M. Lferde "Activities and activity ratios of $\mathrm{U}$ and $\mathrm{Ra}$ radio-isotopes in drinking wells, springs and tap water samples in Morocco". Journal of Radiochemica Acta, 88 (2000) 55-60.

[9] - A. Choukri, 0-K. Hakam, H. Bounouira, M. Al Ibrahimi et J-L. Reyss "Mesure des activités de l'uranium $\left({ }^{238} \mathrm{U},{ }^{234} \mathrm{U}\right)$ et du rapport isotopique ${ }^{234} \mathrm{U} /{ }^{238} \mathrm{U}$ dans des échantillons d'eau naturelle au Maroc à l'aide de la spectrométrie alpha", Afrique SCIENCE, 01(2) (2005) 239-251. http://www.afriquescience.org

[10] - M. Kumar, A.Kumar, S. Singh, R. K. Mahajan \& T. P. S. Walia "Uranium content measurement in drinking water samples using track etch technique" Radiat. Meas., 36 (2003) 479-481.

[11]- M. Doǧru, 0. Baykara, F. Külahcı \& C. Canbazoǧlu "Determination of the radioactivity levels in underground water on some active fault zone of East Anatolian" Balkan Phys. Lett., 11 (2003) 5-8.

[12] - D. Ghosh, A. Deb \& K.K. Patra "Measurements of alpha radioactivity in arsenic contaminated tubewell drinking water using (R-39 detector" Radiat. Meas., 59 (2004) 205-213.

[13] - 0. Baykara \& M. Doǧru "Measurements of radon and uranium concentration in water and soil samples from East Anatolian Active Fault Systems (Turkey)", Radiat. Meas., 41 (2006) 362-367.

[14] - S. Schmidt, J.-L. Reyss "Radium as internal tracer of Mediterranean Outflow Water", Journ. Geophys. Res., 101 (1996) 3589-3596.

[15] - A. Choukri "Application des méthodes de datation par les séries d'uranium à l'identification des hauts niveaux marins sur la côte égyptienne de la mer rouge en moyen de coraux, radioles d'oursins et coquilles, et sur la côte atlantique du Haut Atlas au Maroc, au moyen de coquilles II. Thèse d'État, Univ. Med V, Rabat, (1994) $192 \mathrm{p}$. 